\title{
Definition of the caudate lobe of the liver based on portal segmentation
}

\author{
Masamitsu Kumon ${ }^{1, *}$, Tatsuya Kumon' ${ }^{1}$ Emiko Tsutsui ${ }^{1}$, Chihiro Ebashi', Tsutomu Namikawa², Kyoji Ito ${ }^{3}$, \\ Yoshihiro Sakamoto ${ }^{4}$ \\ ${ }^{1}$ Noichi Central Hospital, Konan, Kochi, Japan; \\ ${ }^{2}$ Department of Surgery, Kochi Medical School, Nankoku, Kochi, Japan; \\ ${ }^{3}$ Department of Hepato-Biliary-Pancreatic Surgery, National Center for Global Health and Medicine, Tokyo, Japan; \\ ${ }^{4}$ Department of Hepato-Biliary-Pancreatic Surgery, Kyorin University Hospital, Mitaka, Tokyo, Japan.
}

\begin{abstract}
Models of liver corrosion were developed by injecting colored Mercox, epoxy resin, silicon rubber and other materials into the portal vein, hepatic artery, bile duct and hepatic vein of autopsied livers. The glissonean or venous branches that obstructed the view of the caudate lobe of the liver were subsequently removed. The detailed anatomy of the caudate vessels was studied and the three parts of the caudate lobe (Spiegel lobe, paracaval portion and caudate process) were defined based on portal segmentation. Caudate portal branches should be defined as dorsal branches arising from the main trunk, or from the first order branches of the portal vein covering the hepatic region in front of the inferior vena cava. The hepatic region, where the internal branches from segment eight cover the front of the inferior vena cava, should be defined as segment eight, and not as the paracaval portion. Prof. Couinaud defined the right side of the caudate lobe as segment IX based on the spatial position; however, this classification of the caudate section seemed to lack consistency with that of other hepatic segments, which were defined based on portal segmentation. We have sustained the dogma that any hepatic segment should be defined based on portal segmentation, and our classification of the definition and boundary of the caudate lobe, which was published in 1985, has sufficient consistency to be used as an international standard.
\end{abstract}

Keywords: caudate lobe, paracaval portion, liver surgery, anatomy of the liver, liver cast, portal segmentation

\section{Introduction}

The caudate lobe of the liver is located deep in the liver, in front of the inferior vena cava (IVC) behind the three major hepatic veins, and cranial to the hilar plate. Thus, the diagnosis and treatment of liver cancer arising in the caudate lobe requires close attention owing to its unique location. However, the definition and boundary of the caudate lobe remains controversial.

The famous anatomist, Prof. Couinaud (1922-2008) classified the liver into eight segments (1); however, he later withdrew his idea that the caudate lobe should be classified into segments I and IX (2). After his death, there has been no international consensus on the definition of the caudate lobe of the liver.

At the beginning of the 1980 's, when anatomical resection of 2-3 sections of the liver was started with acceptable safety, detailed anatomy of the caudate lobe was unclear. At that time, staff surgeons in the Department of Liver Surgery, National Cancer Center Hospital, Tokyo, began to consider that the hepatic area in front of the IVC and the cranial aspect of the portal bifurcation might also belong to the caudate lobe. Knowledge of the caudate lobe anatomy was very limited. One of the authors (MK), who had already started to make liver casts at that time, began to focus on the anatomy of the caudate lobe.

The purpose of the present study was to make a rational definition of the caudate lobe based on portal segmentation, which could be used as an international standard.

\section{Materials and Methods}

The first author (MK) prepared 75 human liver casts between July 1, 1981 and October 2, 1990 (3,4). The candidates included a fetus of the $17^{\text {th }}$ week and old men of uncertain age. The methods used to make casts, including the selection of the injecting materials, were unique to the authors, since there was currently no standard method. There was a wide range of variety in the quality of the casts, and most of them were not suitable for use in further studies; this was primarily because of the fact that the task was difficult for one 
person to perform alone.

Colored resin was injected into the portal vein (blue), hepatic artery (red), bile duct (yellow), and hepatic vein (black) of the whole liver. The specimens were fixed in water and not left on the desk, to preserve the natural hepatic shapes, as they would be inside the body. Although the liver casts-manufactured using Mercox resin were fragile, they were suitable for making casts from fetuses. When an excess of Mercox resin was injected, the hepatic sinusoid overflowed with resin making the casts as hard as stone, and it became impossible to observe the inside of the liver. Liver casts manufactured using silicon rubber were not tolerant of age-associated deterioration for more than 30 years, and many of them were transformed and/or had degenerated (5). Liver casts manufactured using epoxy resin had poor plasticity, were well controlled, and showed no deformities. Of these, we selected several casts with a good shape, an adequate amount of injected resin, a good state of preservation, and those in which the IVC was preserved over a long distance. Then, we dissected the casts intensively, examined the anatomy of the caudate lobe in detail, and took stereoscopic photographs of the liver specimens.

After fixation, the Glissonean and venous branches obstructing the caudate lobe were removed. During the dissection of the liver casts, we used forceps with fine tips and extracted the small Glissonean and venous branches, gently, piece by piece. Large vessels, such as the middle hepatic vein (MHV), were totally or partially resected when they obstructed the view of the deep structure of the liver. After these meticulous steps, we could expose the vascular structure of the caudate lobe and capture stereoscopic photography.

This study was approved by the National Center for Global Health and Medicine Research Ethics Committee/ Institutional Review Board (approval number: NCGM-G-004020-00).

\section{Results}

The anatomy of the caudate lobe was examined and classified into three parts as follows, i) Spiegel lobe, ii) paracaval portion and iii) caudate process. The caudate portal branches should be defined as dorsal branches from the main trunk, or from the first order branches of the portal vein covering the hepatic region in front of the IVC. In cases with a trifurcated type portal vein, the caudate portal branches should be ramified from the main portal trunk of the portal vein, and not from the anterior or posterior portal vein.

\section{Spiegel lobe and the portal branches}

The Spiegel portal branches were defined as dorsal portal branches ramified to the left-side caudate lobe from the main trunk or from the first order portal branches. A relatively large portal branch often ramified from the left portal vein was distributed in the Spiegel lobe. The Arantius ligament lies on the boundary between the left lateral section and the Spiegel lobe, and was easily detected and helpful for dividing the anatomical sections.

\section{Paracaval portion}

The paracaval portal branches were defined as dorsal cranial portal branches ramified from the main trunk, or from the first order portal branches, including branches having a common trunk with Spiegel branches, but excluding ventral branches. The dorsal cranial portal branches from the root of the anterior or posterior portal vein should not be included in the paracaval branches. Our study was conducted based on the concept of portal segmentation.

Defining the boundary between the caudate lobe and the anterior section in a case having an internal branch from segment eight

In a case where an internal branch from segment eight supplied the hepatic region in front of the IVC, we added a detailed dissection of the caudate lobe to reveal the boundary between the paracaval portion and segment eight of the liver. The whole liver was set to observe the paracaval portion from the cranial side (Figure 1A), and the tiny branches around the MHV were removed to reveal the root of the MHV (Figure 1B). The MHV was then divided at its root (Figure 1C). Further dissection of the paracaval portion revealed the main branch in the paracaval portion accompanied by internal branches from segment eight (Figure 1D, Figure 2A). Removal of the tiny branches in the paracaval portion revealed short hepatic veins (SHVs) draining into the IVC and the tips of the caudate process branches (Figure 2B). The cranial aspect of the portal bifurcation was exposed following removal of the caudate lobe of the liver (Figure 2C).

These SHVs were located at the boundary between the paracaval portion of the caudate lobe and segment eight, suggesting that intersegmental hepatic veins can be identified along the boundary among other hepatic segments.

Thus, the right-sided boundary of the paracaval portion of the liver could be defined based on portal segmentation, but not by its spatial position.

\section{Caudate process}

The caudate process is connected to the posterior section of the liver along the Rouviere's sulcus, and the portal branches in the caudate process were always definite. The branches of the caudate process were defined as dorsal caudal portal branches ramified from the main 
How to watch a stereoscopic photography:

1). It is possible to watch the photo sterically with naked eyes using a cross method. 2). When you use the stereoscope to watch the pictures, you may often find left and right reversed images. When the images are reversed, cut off the wave line in the center of the two pictures, and reverse the positions of the two pictures.

A
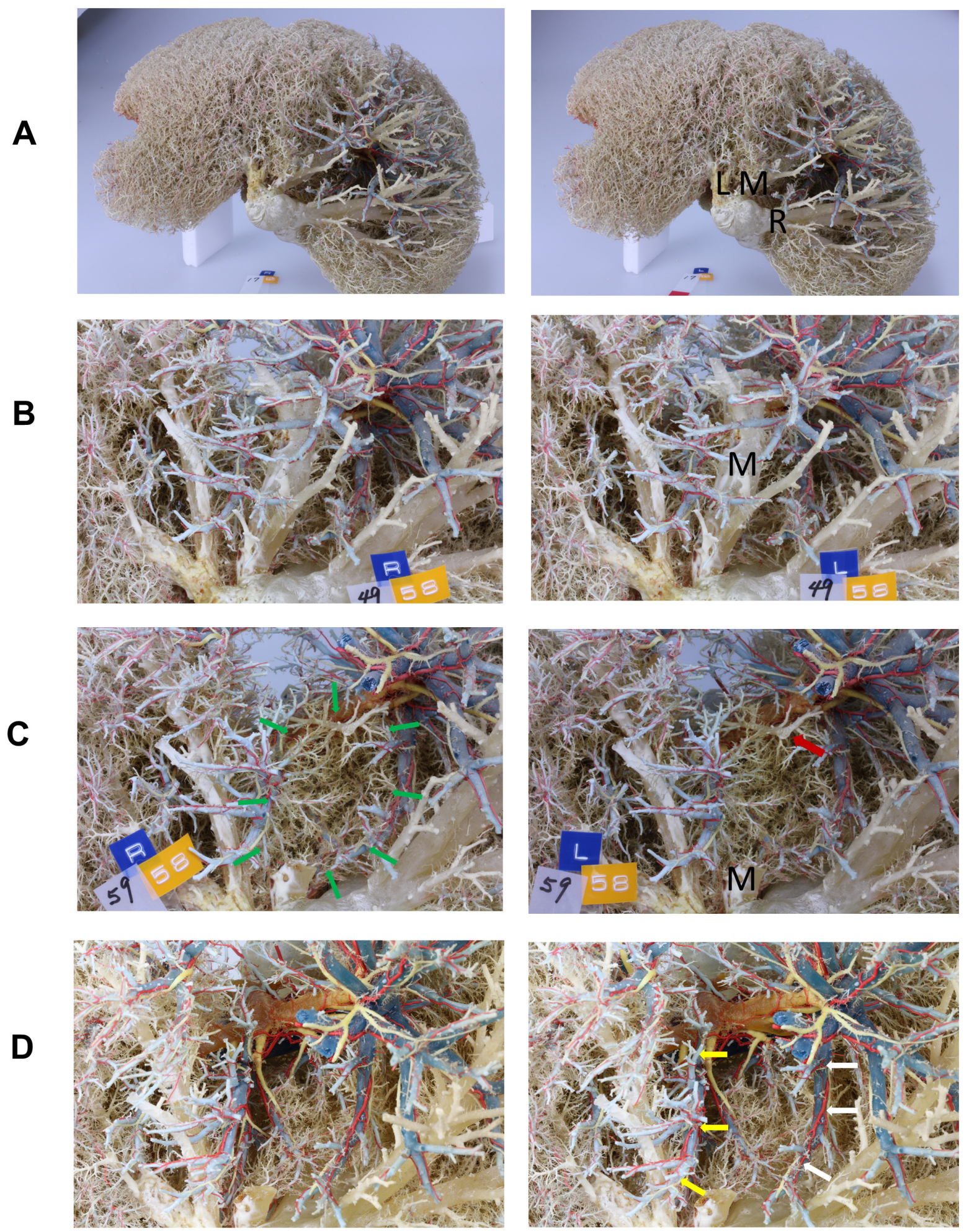

Figure 1. (A) Cranial view of the accomplished liver cast. This view of the cast almost coincides with the intraoperative view of the liver from the position of the anesthesiologists. L, left hepatic vein; M, middle hepatic vein; R, right hepatic vein. (B) Cranial view of the caudate lobe just before the division of the middle hepatic vein. $M$, middle hepatic vein. (C) Cranial view of the caudate lobe just after the division of root of the middle hepatic vein. A numerous number of small caudate branches are seen just behind the middle hepatic vein (green arrows). Red arrow indicates hepatic veins located at the ventral side of the caudate lobe draining into the back of the middle hepatic vein. M, middle hepatic vein. (D) Glissonean branches distributing in the caudate lobe. The peripheral branches obstructing the view have been removed step by step. White arrow indicates the internal branches in the anterior section, and yellow arrow indicates the main trunk of the portal vein in the paracaval portion. 

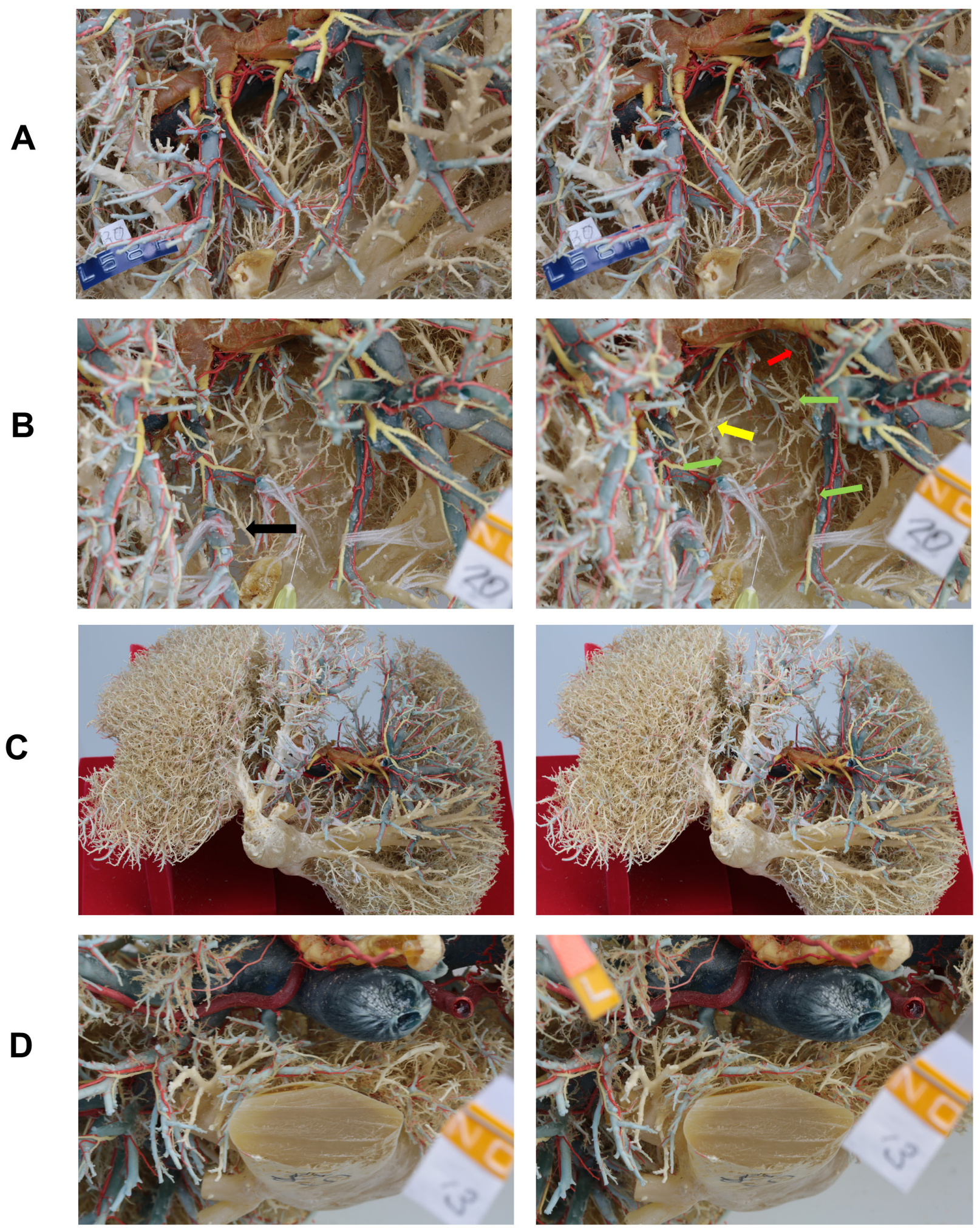

Figure 2. (A) Relationship between the inferior vena cava and the caudate lobe. The outline of the inferior vena cava (IVC) has been gradually exposed. Along with the exposure of the IVC, the cellular space in front of the IVC which was described by Couinaud and colleagues are revealed. This cellular space coincides with the space where a tape is placed to lift the liver during Belghiti's hanging maneuver (18). (B) Cranial view in front of the inferior vena cava. Green arrows indicate the short hepatic veins (SHVs). Yellow arrow indicates the SHV drained from the Spiegel lobe. Black arrow indicated the venous branches draining into the middle hepatic vein. Red arrows indicate the branches in the caudate process. The size of the needle was $30 \mathrm{G}(=300 \mu \mathrm{m})$ and the needle is put on the stump of the middle hepatic vein. (C) View of the bifurcation of the portal vein after complete removal of the caudate branches. Cranial view similar with that in Figure 1A. The cranial aspect of the portal bifurcation can be seen for the whole length. (D) Caudal view of the caudate process. Many communicating veins are seen. 
trunk or from the first order branches of the right portal vein. This is because there were either fewer caudate process branches or many variations in cases with posterior independent type of poral branching (Figure 2D).

\section{Discussion}

In the present study, we clearly defined the caudate lobe of the liver based on portal segmentation. The concept of diving the caudate lobe into three portions may be classical, but there has been no international consensus of the definition on the caudate lobe especially on the right-sided boundary of the paracaval portion of the liver. Our definition of the caudate lobe based on portal segmentation resolves the longtime puzzlement of Prof. Couinaud, and provides a simple and international consensus of the definition on the caudate lobe.

In the history of the anatomical study of the hepatic segments, the caudate lobe was first described as the "lobus exiguous" by Adrian van der Spiegel in 1622. However, the detailed anatomy of the caudate lobe was not revealed until the recent era. The definition and boundary of the caudate lobe differ slightly depending on the researchers and there has been no international consensus on this topic.

\section{Healy and Schroy's caudate lobe}

Healy and Schroy first classified the liver into four sections, the lateral, medial, anterior and posterior segments, and divided each section into cranial and caudal parts, consisting of eight segments (6). They defined the caudate lobe as areas that did not belong to either the left or right liver, and further classified the caudate lobe into three portions, the right portion, left portion, and the caudate process. They studied more than 100 liver casts with cholangiography and demonstrated the paracaval biliary branch, which was named the right caudate duct. They also named the Spiegel branch as the left caudate duct.

\section{Change in the definition of Couinaud's caudate lobe}

Prof. Couinaud classified the liver into eight segments from segment I to VIII, in 1954 (1), and this classification has gradually become more common since the development of hepatobiliary surgery. In 1954, he named the dorsal liver as segment I, and in 1989, he divided segment I into segment Il and segment Ir (8) (Figure 3A); however, in 1994, he replaced segment Ir with segment IX. By this time, he proposed the idea of dividing segment IV into subsegments b, c and d, but the concrete spatial areas of these subsegments were not drawn on the axial schema (8) (Figure 3B). In 1998, he showed a schematic view of subsegments $b$, $\mathrm{c}$, and $\mathrm{d}$ on the axial view of the liver (9) (Figure 3C).

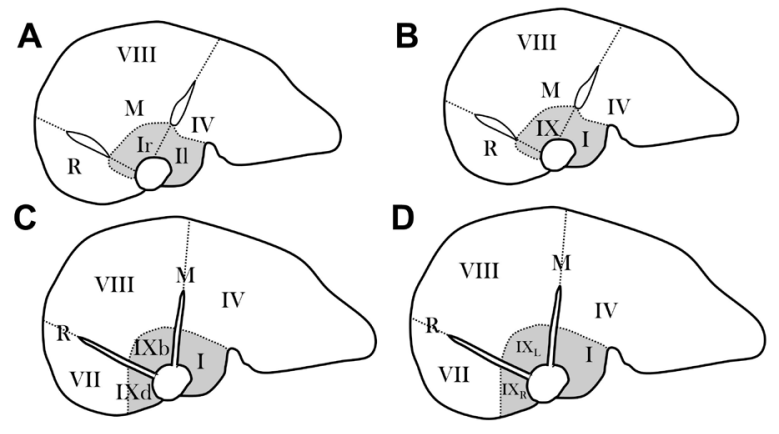

Figure 3. Historical change of definition of the caudate lobe by Prof. Couinaud. (A), In 1989, the caudate lobe was divided into segments Il and Ir based on the spatial position. The boundary between the two segments is the plane connecting middle hepatic vein and inferior vena cava (7). By this time, Couinaud had proposed the idea to divide segment Ir into subsegments b, c and d, but the schema of segmentation was not shown. (B), In 1994, segment Ir was replaced by segment IX (8). By this time, Couinaud had proposed the idea to divide segment IX into subsegments $b, c$ and $d$, but the schema of segmentation was not shown. (C), In 1998, the subsegments of IX were shown on the figure. The right hepatic vein runs between subsegments $\mathrm{b}$ and $\mathrm{d}(9)$. Subsegment IXc is located beneath the right hepatic vein. (D), In 2000, the subsegments $\mathrm{b}$ and $\mathrm{d}$ were replaced by subsegments IXL and $\mathrm{IX}_{\mathrm{R}}(10)$. The figures were modified from the original ones.

In a manuscript by Filipponi, he changed segment IXb and segment IXd to segment IXL and segment IXR, respectively, while maintaining the concept of segments I and IX (10) (Figure 3D). Finally, he abandoned the concept of segment IX in the manuscript by Abdalla in $2002(2)$.

\section{Paradox of Couinaud's caudate lobe}

As is apparent from the transition of the nomenclature and definition of the caudate lobe by Prof. Couinaud, it was difficult to define the range of the caudate lobe, especially on the right-sided boundary. Prof. Couinaud himself noted the following comments in his book of surgical anatomy (1): On the right, in front of the IVC, the exact nature of the pedicles is difficult to state. Are they caudate veins? Do they belong to the right liver? How far does the caudate lobe extend to the right? After a thorough investigation of the right portion of the caudate lobe and, the posterior extremity of segments VII and VIII, a special territory was observed in front of the IVC, posterior to the right portal pedicle, inferior to the plane right superior-middle hepatic veins. This territory is supplied by small and ascending posterior branches of various origins, extending to the right territory of the caudate veins, but is obviously different from the caudate lobe.

This led to the concept of a posterior or dorsal liver (designated as sector I) with a left dorsal segment (caudate lobe or segment Il) and a right dorsal segment (or segment Ir). These designations replace the former segment I. 

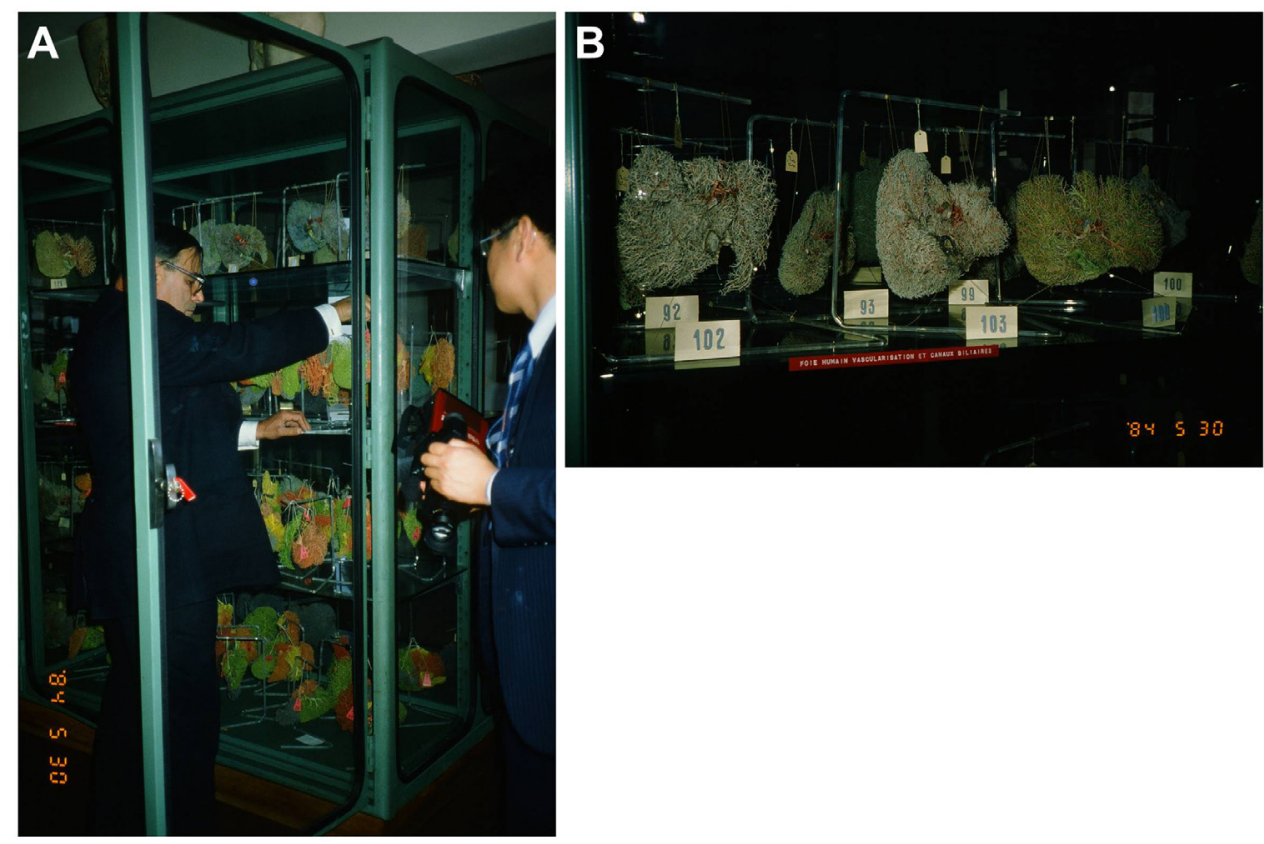

Figure 4. Memorial photos of Prof. Couinaud and his liver casts. (A), Prof. Couinaud gave us the explanation about liver casts of a various animals at the museum of which he played the director. Dr. Nakatani listens to him. (Department of Surgery, Shinshu University, at that time. This picture was taken by KM.) (B), Liver cast manufactured by Prof. Couinaud. The liver cast is compressed back and forth, which suggests that the cast may be manufactured on flat plates.

\section{Discussion with Prof. Couinaud}

We fully understand why Prof. Couinaud found defining the boundary of the caudate lobe so difficult after observing plenty of small vessels in the caudate lobe (Figure 1C). We removed all of the Glissonean branches and hepatic veins obstructing the anatomy of the caudate lobe, and made sure to carefully observe the caudate branches in the hepatic casts. We believe that detailed observation of the roots of the small caudate branches off the first order branch of the portal vein, and the tiny branches covering the caudate lobe would be impossible until we could achieve the abovementioned meticulous dissection.

The first author (MK) remembers that Couinaud's cast (Figure 4) seemed to be manufactured with less resin than that used in their own casts, although the final appearance was quite similar. This suggests that the difference in the definition of the caudate lobe between the two researchers is due to the difference in whether the obstructing hepatic parenchyma and vessels were removed or not.

The first author (MK) asked Prof. Couinaud directly about the right-sided margin of the caudate lobe, at which time, his answer was "I have no answer". He did not comment on the invisible area of the caudate lobe because then, he had no method to observe the vessels that were deeply located in the caudate lobe. We assume that he not only adopted the typical portal branching pattern but also the four atypical variations of the portal branching to the dorsal liver (segment $\mathrm{IX}_{\mathrm{L}}$ and $\mathrm{IX}_{\mathrm{R}}$ ). His classification might mislead the researchers that there were four types of portal branches, and that the caudate portal branches always reached the level of the roots of the right and middle hepatic veins running along the IVC. His schema of the caudate lobe presenting the bifurcation of the caudate branches from the portal vein appears unclear; this suggests that it is important to remove the obstructing branches other than those of the caudate lobe, to grasp the bifurcation pattern of the caudate lobe located in the deepest parts of the liver casts. In MK's casts, no branches were found to be running very close to the IVC in the caudate lobe (Figure 1D, Figure 2A).

\section{Definition of the caudate lobe by other researchers}

Prof. Nimura is a world authority in the treatment of perihilar cholangiocarcinoma, and first advocated the importance of combined resection of the caudate lobe to increase the curability for the surgical treatment of perihilar cholangiocarcinoma. He stated that the concept of Couinaud's segment IX is difficult to understand, and instead defined the caudate lobe as follows (11): The left caudate lobe should be located on the left side of the Arantius ligament between the root of the left hepatic vein and the umbilical portion, while the right caudate lobe should be located on the right side of the left caudate lobe in front of the IVC, and on the left side of the root of the right posterior portal vein (P-point). The tip of the right caudate lobe extends the phrenic surface of the liver between the middle 
and right hepatic veins. The caudate process is the protruding portion of the liver caudal to the right portal vein.

Prof. Nimura's definition of the caudate lobe is similar to that of Couinaud's. Prof. Nimura states that the starting point of the caudate lobe should be determined first, followed by the corresponding spatial area of the caudate lobe. This concept is not based on portal segmentation.

Ryu and Kogure (12) have published many papers, but it seems that they did not define the anatomical boundary of the caudate lobe.

Takayama primarily defined the caudate lobe mostly based on the definition proposed by Couinaud (13), and later became the first surgeon to successfully perform isolated caudate lobectomy without dividing other hepatic segments (high dorsal resection). To this end, he injected blue dye into the liver parenchyma in the caudate lobe, beside the right and middle hepatic veins (tattooing) and determined the right-sided or ventral boundary of the caudate lobe with an aid of the pooled dye. He also injected the dye in the posterior portal branch and stained the posterior section of the liver; this allowed visualization of the boundary between the posterior section and the caudate lobe, and was named as the "counter-staining technique". However, this technique was not used to visualize the boundary between the anterior section and caudate lobe; if the counter-staining were to be used to visualize the ventral boundary of the caudate lobe, it would be more useful. In this manner, the area supplied by the inner branches of the anterior portal vein would be visualized, which would prove that these branches belong to the anterior section, and not to the caudate lobe. Visualization of the right-sided boundary of the caudate lobe using the counter-staining technique should be based on the concept of portal segmentation, which is incompatible with Couinaud's segmentation of the caudate lobe. Notably, none of the above studies on the caudate lobe have been against Couinaud's segmentation.

At the beginning of the 1990's, Prof. Makuuchi described that the caudate lobe only indicated the Spiegel lobe of the liver, missing the caudate process and paracaval potion, in other countries (14).

Importance of definition of the caudate lobe based on portal segmentation and the difficulties of Couinaud's definition

In 1985, we defined that dorsal cranial branches derived from the main trunk or from the first order branch of the poral vein as paracaval branches. In addition, the portal venous branches that ramified from the anterior portal branch supplying the liver parenchyma surrounded by the roots of the right and middle hepatic veins were defined as portal branches in segment eight.

The concept of classifying the caudate lobe into three portions has been accepted in many papers as referred to by Abdalla \& Couinaud (2). However, our idea that the liver area supplied by the branches from the anterior portal vein should be defined as part of the anterior section, and not of the caudate lobe has never been sited or understood.

In the current study, we carefully dissected the liver casts by extracting the small peripheral Glissonean and hepatic venous branches obstructing the caudate. Several questions relating to Couinaud's definition of the caudate lobe were raised as a result of the findings obtained from the dissection of the caudate lobe using our method.

1). The extent of the caudate lobe varied according to the definition of the caudate lobe. For example, "behind the right hepatic vein" might suggest the ventral boundary of the right portion of the caudate lobe, but it was unclear whether this was the main trunk, or the center, right edge or left edge of the right hepatic vein.

2). Prof. Couinaud introduced the concept of the dorsal liver based on the spatial region, and not on portal segmentation; however, he classified other segments (segments II-VIII) based on portal segmentation. Thus, his methods lacked consistency.

$3)$. The nomenclature of the portal branches was complicated and confusing. i) Prof. Couinaud declared that the nomenclature of the portal vein was defined based on the distribution to each segment, and not on the origins of the ramification from the main portal trunk. For example, an internal portal branch belonging to segment seven could be named as "caudate portal branch" or "internal portal branch of segment seven". ii) Prof. Couinaud stated that each portal branch could "borrow" a name depending on the situation. However, it was unclear how the name was "borrow" from other segments. From the route of each portal segment? Or did it "borrow" the name when it entered the caudate area, while also keeping the original branching name? Or did it restore the original name when it went out of the caudate area? This was unclear. Cho (15), Matsui (16), and Maki (17) performed a meticulous and detailed analysis of the imaging study and reported that the peripheral branches of the caudate lobe could reach the phrenic surface of the liver. How would Prof. Couinaud name these peripheral portal branches?

4). Prof. Couinaud defined the dorsal liver as consisting of $i$ ) the Spiegel lobe, $i i$ ) the paracaval lobe and $i i i)$ the caudate process. This classification perfectly coincides with that of Kumon; thus, we believe that Prof. Couinaud understood and accepted Kumon's classification. However, Prof. Couinaud abandoned segment IX which he advocated. Namely, he found the paradox of his theory and accepted Kumon's classification.

Herein, we have listed the above four paradoxes of Couinaud's theory. We cannot and should not accept his 
definition of the caudate lobe as it stands. It is required to establish an international standard definition of the caudate lobe as soon as possible.

Toward an international definition of the caudate lobe of the liver

The author (MK) first proposed the anatomical definition and boundary of the caudate lobe of the liver in 1985, and Couinaud accepted the rational of our concept, as described above. Couinaud's classification has contradictions and cannot be used as a world standard. Although Nimura's definition is fundamentally similar to ours, it is not based on the concept of portal segmentation. Kumon's definition of the caudate lobe published in 1985 is based on the concept of portal segmentation, and is both simple, and easy to understand. Thus, this definition can be used as the world standard in clinical settings, because the landmark and boundary of the caudate lobe is described clearly, and will help to elucidate the right-sided boundary of the caudate lobe in the future.

It is possible that some researchers may be worry if the definition of the caudate lobe is settled by our concept, as opposed to the traditional definition outlined by Prof. Couinaud's. However, we believe that a solution is possible if an internationally unified definition is set up with sufficient evidence.

\section{Conclusion}

We studied the vascular and biliary structures of the caudate lobe using liver casts to elucidate the definition of the caudate lobe. Since we could observe the whole circumference of the left and right portal vein, and the first order brances of the portal vein, we could resolve some inconsistencies that Prof. Couinaud could not. We also found that the definition of the caudate lobe by Prof. Couinaud was fraught with issues, and consequently, we determined that a new definition was required. Kumon's classification is based on portal segmentation, and we believe that it has the potential to be easily accepted internationally. However, the proposal of a new definition of the caudate lobe will inevitably be met with opposing opinions, since the highly regarded Prof Couinaud has proposed and applied the concept of the dorsal liver to the caudate lobe. However, let us make an international and simple definition of the caudate lobe beyond these opinions. We aim to show the outline of the caudate lobe according to the types of portal ramification in a future issue, with the aim to further understand and spread the anatomy of the caudate lobe.

\section{Acknowledgments}

We would like to express our gratitude to Prof. Norihiro
Kokudo, President of National Center for Global Health and Medicine for his helpful advice and kindness. He also supported the first author (MK) with the English publication of his original work $(2,3)$. We also express our gratitude to Prof. Kazuhiro Hanazaki, Department of Surgery, Kochi Medical School for his kind contribution to our study.

\section{Funding: None.}

Conflict of Interest: The authors have no conflict of interest to disclose.

\section{References}

1. Couinaud C. Lobes et segments hépatiques: notes sur l'architecture anatomiques et chirurgicale du foie (Liver lobes and segments: notes on the anatomical architecture and surgery of the liver). Presse Med. 1954: 62:709-712. (in French)

2. Abdalla EK, Vauthey JN, Couinaud C. The caudate lobe of the liver. Implications of embryology and anatomy for surgery. Surg Oncol Clin N Am. 2002; 11:835-848.

3. Kumon M. Anatomy of the caudate lobe with special reference to portal vein and bile duct. Acta Hepatol Jpn 1985; 26:1193-1199. (in Japanese)

4. Kumon M. Anatomical study of the caudate lobe with special reference to portal venous and biliary branches using corrosion liver casts and clinical application. Liver Cancer. 2017; 6:161-170.

5. Kumon M, Ogata T, Nakatani Y, Hasegawa H. Moules hépatiques souples en silicone (Flexible silicone hepatic casts). Ann Chir. 1985; 39:221-223. (in French)

6. Healey JE, Schroy PC. Anatomy of the biliary ducts within the human liver. Arch Surg. 1953; 66:599-616.

7. Couinaud C. Surgical anatomy of the liver revisited. Acheve Dimprimer Sur Les Presses, Paris, 1989; pp 130132.

8. Couinaud C. The paracaval segments of the liver. J Hepatobiliary Pancreat Surg. 1994; 2:145-151.

9. Couinaud C. Dorsal sector of the Liver. Chirurgie. 1998; 123:8-15.

10. Filipponi F, Romagnoli P, Mosca F, Couinaud C. The dorsal sector of human liver: embryological, anatomical and clinical relevance. Hepatogastroenterology. 2000; 47: 1726-1731.

11. NimuraY. Surgical Anatomy of the Caudate Lobe Segment I and Segment IX -. Tan To Sui. 1996; 17:11891196. (in Japanese)

12. Kogure K, Kuwano H, Fujimaki N, Makuuchi M. Relation among portal segmentation, proper hepatic vein, and external notch of the caudate lobe in the human liver. Ann Surg. 2000; 231:223-228.

13. Akayama T, Tanaka T, Higaki T, Katou K, Teshima Y, Makuuchi M. High dorsal resection of the liver. J Am Coll Surg. 1994; 179:72-75.

14. Makuuchi M. Why is resection of the caudate lobe of the liver necessary? Geka. 1996; 58:387-391. (in Japanese)

15. Cho A, Okazumi S, Makino H, et al. Vessels of the Caudate Lobe and Hilar Plate. Tan To Sui. 2003; 24:6773. (in Japanese)

16. Matsui O, Takashima T, Kadoya M, Hirose J, Kameyama 
T, Choto S, Konishi H. (CT anatomy of para-caval portion of the caudate lobe of the liver). Nihon Igaku Hoshasen Gakkai Zasshi. 1988;48:841-846. (in Japanese)

17. Maki H, Sakamoto Y, Kawaguchi Y, et al. Anatomical Boundary Between the Caudate Lobe of the Liver and Adjacent Segments Based on Three-Dimensional Analysis for Precise Resections. J Gastrointest Surg 2018; 18:1709-1714.

18. Belghiti J, Guevara OA, Noun R, et al. Liver hanging maneuver: a safe approach to right hepatectomy without liver mobilization. J Am Coll Surg. 2001; 193:109-111.
Received October 14, 2020; Revised October 20, 2020; Accepted October 24, 2020.

Released online in J-STAGE as advance publication October 26, 2020.

*Address correspondence to:

Masamitsu Kumon, Noichi Central Hsopital, 555-18 Higashino, Noichi, Konan, Kochi 781-8213, Japan.

E-mail:kumon@kouseikai-noichi-ch.or.jp 